\title{
Different morphologies of polyaniline nanostructures synthesized by interfacial polymerization
}

\author{
Tiago V. Freitas ${ }^{a}$, Eliraldrin A. Sousa ${ }^{\mathrm{b}}$, Gilberto C. Fuzari Jr ${ }^{\mathrm{a}}$, Elen P.S. Arlindo ${ }^{\mathrm{a}, *}$ \\ a Instituto de Ciências Exatas e da Terra, Universidade Federal de Mato Grosso, CEP 78600-00 Barra do Garças, MT, Brazil \\ ${ }^{\mathrm{b}}$ Departamento de Física e Química, Universidade Estadual Paulista, CEP 15385-000 Ilha Solteira, SP, Brazil
}

\section{A R T I C L E I N F O}

\section{Article history:}

Received 31 January 2018

Received in revised form 11 April 2018

Accepted 14 April 2018

Available online 15 April 2018

\section{Keywords:}

Polyaniline

Interfacial polymerization

Nanofibers

Nanosheets

Nanoflowers

\begin{abstract}
A B S T R A C T
In this study, different morphologies of polyaniline (PAni) nanostructures were obtained via interfacial polymerization. The influence of the time and temperature of polymerization on morphology was investigated. At room temperature the growth of nanosheets and nanoflowers with long polymerization time (1-5 days) was observed. In the syntheses carried out at $50{ }^{\circ} \mathrm{C}$ the same nanostructures were obtained with only a few hours of polymerization, which illustrates that the increase in temperature considerably reduced the synthesis time and also provided the growth of nanofibers, morphology not found in the syntheses performed at room temperature. The characterization by XRD indicated the presence of diffraction peaks characteristic of PAni nanostructures with high crystallinity. As the conductivity of PAni is directly related to its crystallinity, it is expected that the nanostructures obtained have high conductivity, which may represent a greater potential for diverse applications. It can be concluded that the proposed synthesis presents a simple alternative for obtaining PAni nanostructures with different morphologies and high crystallinity.
\end{abstract}

(c) 2018 Elsevier B.V. All rights reserved.

\section{Introduction}

Polyaniline (PAni) is a conductive polymer with good processability, chemical stability, and low cost. It can be used in various applications, such as rechargeable batteries, electromagnetic radiation shielding, field-effect transistors, gas sensors, and memory devices [1-4].

Usually, PAni can be synthesized from the aniline monomer by chemical or electrochemical polymerization. Several chemical methods for obtaining PAni nanostructures, such as interfacial polymerization, which occurs in a two-phase system formed by an organic solvent and an aqueous phase, have been reported [5-7].

In recent years, several morphologies of PAni nanostructures have been reported, including nanofibers [5,6,8-14], nanosheets $[9,15,16]$, and nanoflowers [7,15,17-21]. These nanostructures have been widely studied because of their promising application in nanomaterials and nanodevices $[5,9,15]$. Some of these morphologies, in general, have high crystallinity and consequently higher conductivity, which may further increase their application potential.

\footnotetext{
* Corresponding author.

E-mail address: elenpoliani@yahoo.com.br (E.P.S. Arlindo).
}

The main goal of this work is to investigate the influence of the polymerization time and temperature on the morphology of PAni nanostructures synthesized using interfacial polymerization.

\section{Experimental}

PAni was synthesized using the interfacial polymerization method. Two solutions were prepared, as follows: aqueous (ammonium persulfate $+\mathrm{HCl}$ ) and organic solution (distilled aniline + toluene). It was initially fixed in the organic solution, with $2 \mathrm{~mL}$ of aniline and $10 \mathrm{~mL}$ of toluene, and in the aqueous solution, it was fixed with $100 \mathrm{~mL}$ of $1.0 \mathrm{M} \mathrm{HCl}$. The amount of ammonium persulfate (oxidant) for achieving a 1:1 $\mathrm{M}$ ratio of aniline:oxidant was determined. Following this, the organic solution was slowly added to the aqueous solution for $30 \mathrm{~min}$. Among the organic and aqueous solutions an interface is formed that has the characteristics to initiate the PAni polymerization process: aniline, oxidant and acid solution. The resulting PAni is rapidly decanted into the aqueous solution. After the polymerization was complete, the toluene was removed, and then the remaining solution was filtered and washed, first in $0.1 \mathrm{M} \mathrm{HCl}$ solution and then in acetone. The resulting PAni powder, obtained as the emeraldine salt form, was dried at $60{ }^{\circ} \mathrm{C}$ for $24 \mathrm{~h}$. Table 1 shows the varied parameters in each synthesis. 
Table 1

Polymerization Parameters Used in Each Synthesis.

\begin{tabular}{|c|c|c|c|c|c|c|c|c|}
\hline & PAni 1 & PAni 2 & PAni 3 & PAni 4 & PAni 5 & PAni 6 & PAni 7 & PAni 8 \\
\hline Temperature $\left({ }^{\circ} \mathrm{C}\right)$ & 25 & 25 & 25 & 25 & 50 & 50 & 50 & 50 \\
\hline Time (h) & 1 & 24 & 48 & 120 & 1 & 2 & 5 & 12 \\
\hline
\end{tabular}

The morphological characterization was then performed using a scanning electron microscope (SEM, Zeiss, model: Evo LS-15) and field emission gun SEM (FEG-SEM, JEOL, model: JSM-7100FT). Xray diffraction (XRD) characterization was performed using a Bruker D8 Discover diffractometer with $\mathrm{Cu} \mathrm{K} \alpha$ radiation in the range of 5 to $30^{\circ}$, with steps of $0.02^{\circ}$, under a rotation of $15 \mathrm{rpm}$ during the measurement.

\section{Results and discussion}

Fig. 1a illustrates the PAni 1 image; slightly elongated structures can be observed, from which it is assumed that the polymer- ization time was not sufficient for the formation of nanofibers. Fig. 1b (PAni 2) shows the formation of nanosheets, as observed by Shendkar et al. [9] and Tao et al. [15,16]; the longer polymerization time $(24 \mathrm{~h})$ contributed to the formation of this morphology. In Fig. 1c for PAni 3, the image illustrates nanosheet agglomerates, and it can be concluded that the longer polymerization time favored the formation of these agglomerates. In the PAni 4 image (Fig. 1d), the appearance of nanoflowers can be noticed, as observed by Yang et al. [18] and Sun et al. [19] and described by Wang et al. [21] as a random nanosheet arrangement. In the syntheses carried out under a temperature of $50^{\circ} \mathrm{C}$, it can be seen in PAni 5 (Fig. 1e) that with $1 \mathrm{~h}$ of polymerization, nanofiber growth is observed, indicating that the increase in temperature accelerates
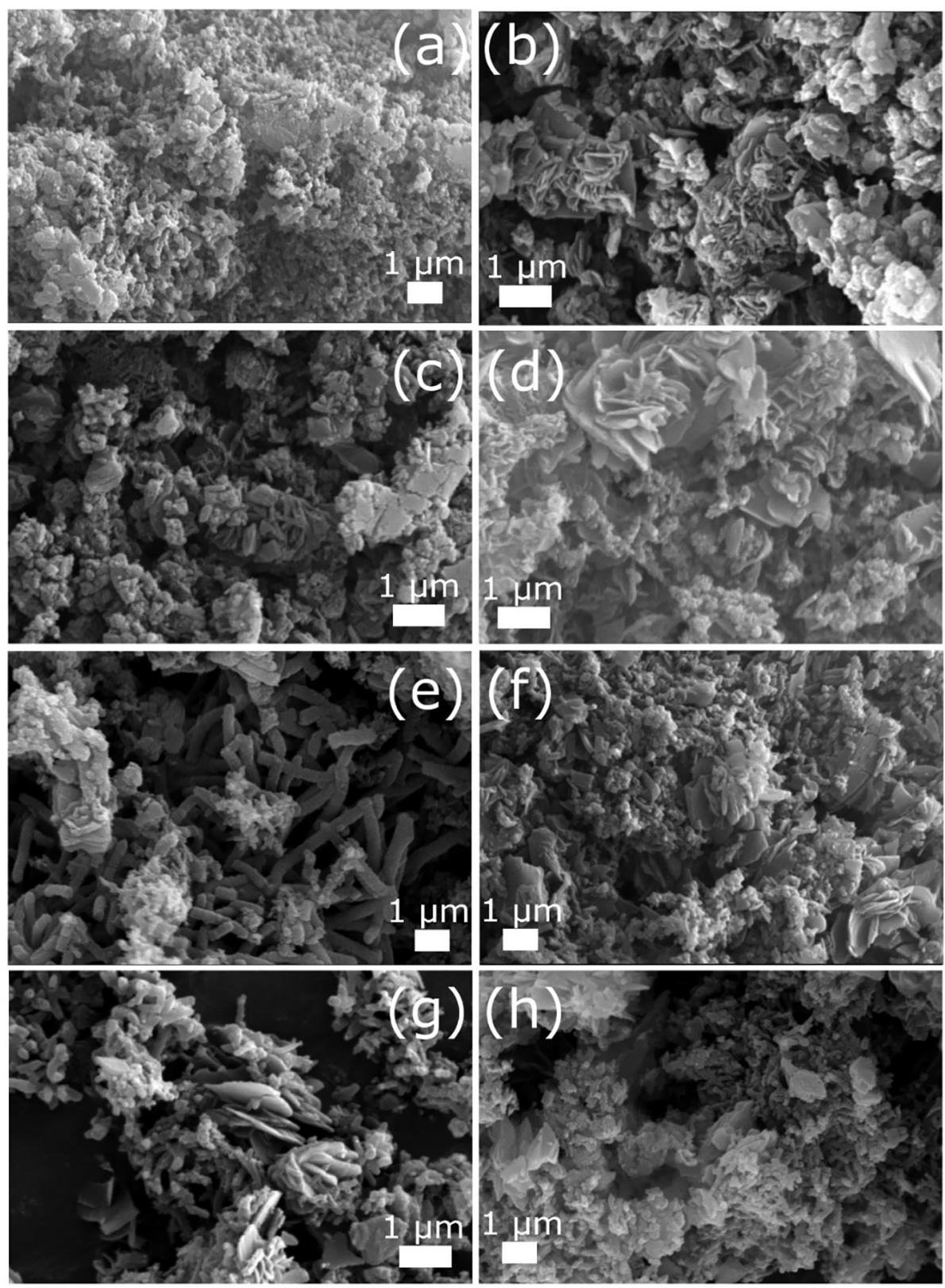

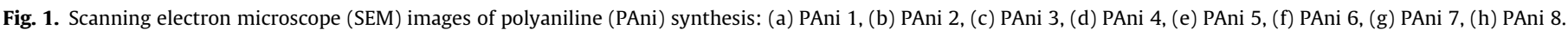




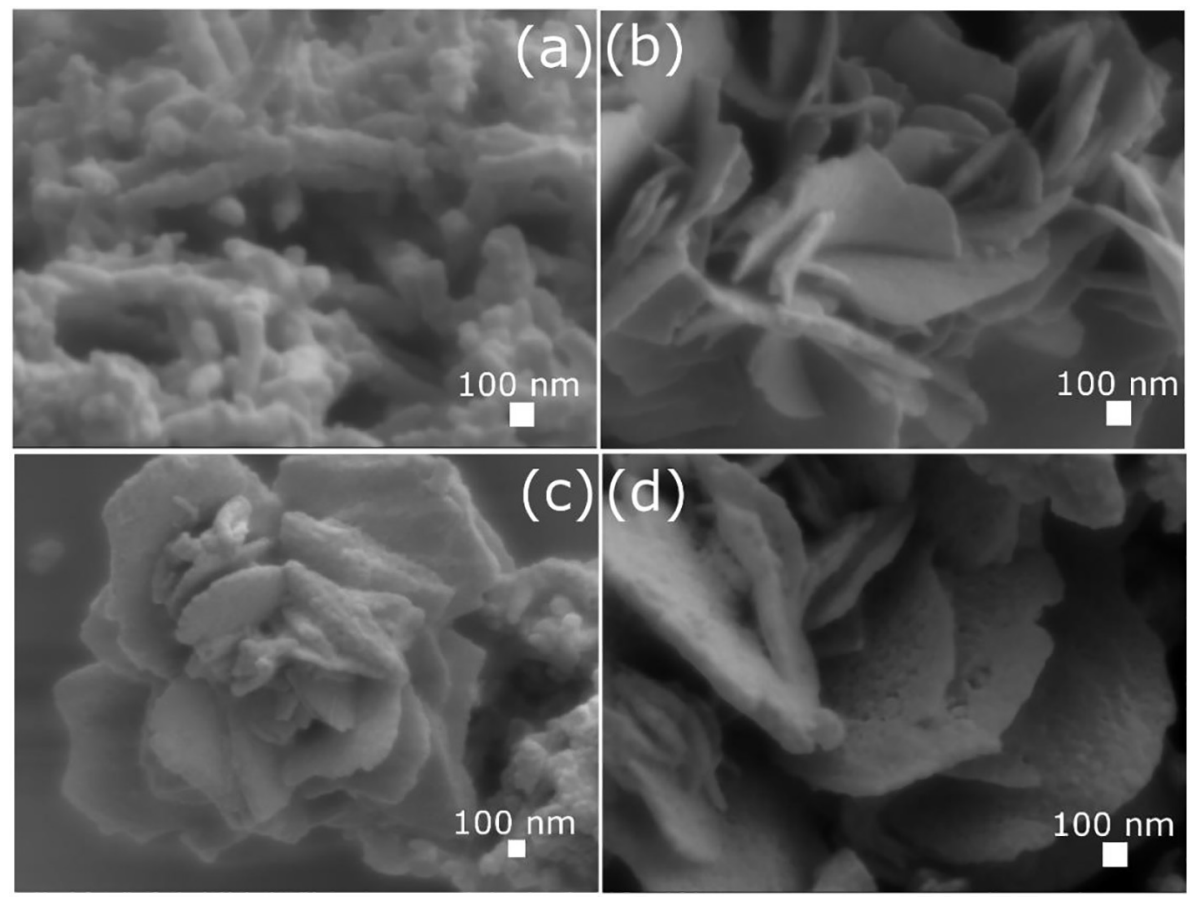

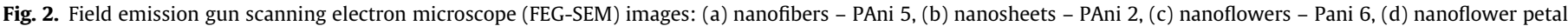
detail - PAni 6.

the polymerization process, thereby favoring the growth of this type of morphology. At $25^{\circ} \mathrm{C}$, with the same polymerization time, it was not possible to observe the formation of nanofibers. In the PAni 6 image (Fig. 1f), it is again possible to observe the temperature influence on the nanostructure morphology, with the nanoflowers obtained with only $2 \mathrm{~h}$ of polymerization. In the PAni 7 image (Fig. 1g), nanosheet and plate structures are again obtained, representing an ordered nanosheet arrangement; here, thicker sheets were formed, as proposed by Wang et al. [21]. In PAni 8 (Fig. $1 \mathrm{~h}$ ), the predominance of structures with a plate morphology can be observed, indicating that the longer polymerization time favors the sheets' ordered growth, thereby forming the plates.

Fig. 2 illustrates the FEG-SEM images of some of the synthesized morphologies. In Fig. 2a, it is possible to observe various nanofibers with a diameter of approximately $150 \mathrm{~nm}$. Fig. 2b shows some nanosheets with a thickness of around $100 \mathrm{~nm}$. Fig. 2c and 2d provide detailed images of a nanoflower resulting from the nanosheets' random stacking.

Fig. 3 presents the XRD pattern of the synthesis from PAni 1 to PAni 8. All syntheses present the characteristic peaks of the emeraldine salt form at $9.2^{\circ}, 14.9^{\circ}, 20.6^{\circ}$, and $25.4^{\circ}$ [22]. The peaks at $6.4^{\circ}, 18.2^{\circ}, 19.2^{\circ}$, and $22.9^{\circ}$, which are characteristic of PAni nanostructures with high crystallinity [23], were observed only in the PAni 2-8 syntheses. A diffraction peak at $6.4^{\circ}$ represents the periodical distance between the dopant and $\mathrm{N}$ atom on the adjacent main and characterizes the greater linearization of the polymeric chain that favors electrical conductivity [22-24]. The appearance of this peak may have been favored by the dropwise addition of the reagent, since the slow addition may favor a greater organization of the polymer chains. In the synthesized materials, the peak is more intense in the syntheses in which the nanosheets are present in greater quantity, and it continues to increase as the nanoflowers

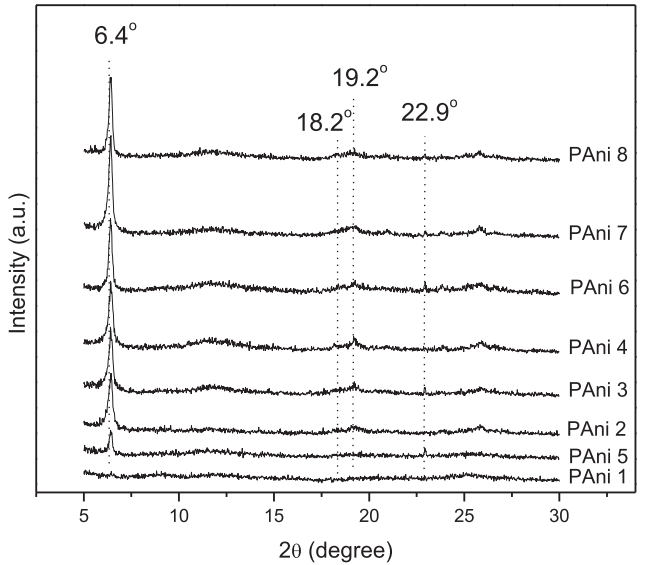

(a)

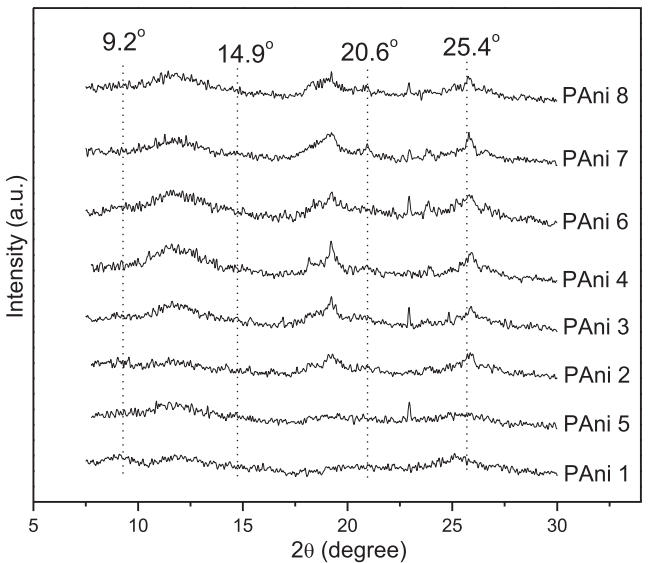

(b)

Fig. 3. X-ray diffraction (XRD) patterns of the synthesis in the ranges of (a) $5-30^{\circ}$, (b) $7.5-30^{\circ}$. 
and plates appear. As mentioned above, the appearance of this peak is directly related to the PAni conduction, so it is expected that these nanosheets, nanoflowers, and plates will have high conductivity.

As shown above, interfacial polymerization proves to be an effective method for obtaining different morphologies PAni nanostructures, using only the influence of polymerization time and temperature. Temperature is shown to be a significant factor in reducing the synthesis time, since nanostructures with nanoflower morphologies were obtained at $50{ }^{\circ} \mathrm{C}$ with only $2 \mathrm{~h}$ of polymerization. The same morphology was obtained by Wang et al. [21] between 1 and 3 days of synthesis. In addition to the decrease in synthesis time in relation to the syntheses reported for this morphology, the nanosheets and nanoflowers obtained in the present work present higher crystallinity, which may represent higher conductivity, and therefore, greater potential for diverse applications.

\section{Conclusions}

5. In this study, different PAni nanostructures were obtained via interfacial polymerization (nanofibers, nanosheets, and nanoflowers). At room temperature, a longer polymerization time led to the formation of nanosheets and nanoflowers. The same process occurred at a temperature of $50{ }^{\circ} \mathrm{C}$, however the increase in temperature accelerated the polymerization process, allowing the growth of these morphologies with shorter polymerization times and also provided the growth of other morphologies such as nanofibers and plates, which were not observed in the syntheses performed at room temperature. The XRD analysis indicated that the nanosheets and nanoflowers had structures with high crystallinity, which can significantly increase the conductivity of the polymer. It can be concluded that the proposed synthesis presents a simple alternative for the control of different morphologies with high crystallinity in PAni nanostructures.

\section{Acknowledgement}

We would like to acknowledge the financial support of the Brazilian funding agency FAPEMAT. The SEM facilities were provided by DFQ-FEIS-UNESP, while the FEG-SEM and XRD facilities were provided by CRTI-UFG.

\section{References}

[1] A. Rahy, D.J. Yang, Mater. Lett. 62 (2008) 4311-4314.

[2] G. Wang, S. Zhuo, W. Xing, Mater. Lett. 69 (2012) 9-27.

[3] M. Hughes, G.Z. Chen, M.S.P. Shaffer, D.J. Fray, A.H. Windle, Chem. Mater. 14 (2002) 1610-1613.

[4] S. Bhadra, D. Khastgir, N.K. Singha, J.H. Lee, Prog. Polym. Sci. 34 (2009) $783-$ 810.

[5] F. Zeng, Z. Qin, B. Liang, T. Li, N. Liu, M. Zhu, Prog. Nat. Sci-Mater. 25 (2015) 512-519.

[6] R. Li, Z. Chen, J. Li, C. Zhang, Q. Guo, Synth. Met. 171 (2013) 39-44.

[7] B. Rajender, S. Palaniappan, Int. J. Polym. Mater. Po. 64 (2015) 939-945.

[8] L.R. Oliveira, L. Manzato, Y.P. Mascarenhas, E.A. Sanches, J. Mol. Struct. 1128 (2017) 707-717.

[9] J.H. Shendkar, N. Zate, K. Tehare, V.V. Jadhav, R.S. Mane, M. Naushad, J.M. Yun, K.H. Kim, Mater. Chem. Phys. 180 (2016) 226-236.

[10] H. Xu, X. Li, G. Wang, J. Power Sources 294 (2015) 16-21.

[11] S. Chuanyu, M.D.F. Rahman, W.Yu. Optoelectron, Adv. Mat. 8 (2014) 810-813.

[12] S. Dutt, P.F. Siril, J. Appl. Polym. Sci. 131 (2014) 40800.

[13] P. Chutia, A. Kumar, Polym. Eng. Sci. 55 (2015) 995-1002.

[14] H. Fan, N. Zhao, H. Wang, X. Li, J. Xu, Mater. Lett. 78 (2012) 42-45.

[15] Y. Tao, F. Wang, N. Peng, S. Wang, G. Ding, G. Cheng, Russ. J. Phys. Chem. A 89 (2015) 1449-1451.

[16] Y. Tao, G. Cheng, M. Zhang, L. Hu, Q. Yu, G. Ding, Russ. J. Phys. Chem. A 89 (2015) 2267-2270.

[17] C. Zhou, X. Gong, Y. Qu, J. Han, Appl. Surf. Sci. 379 (2016) 124-131.

[18] M. Yang, Z. Xiang, G. Wang, J. Colloid Interf. Sci. 367 (2012) 49-54.

[19] J. Sun, H. Bi, Appl. Surf. Sci. 258 (2012) 4276-4282.

[20] W. Zhong, Y. Li, Y. Wang, X. Chen, Y. Wang, W. Yang, J. Colloid Interf. Sci. 365 (2012) 28-32.

[21] Y. Wang, J. Liu, H.D. Tran, M. Mecklenburg, X.N. Guan, A.Z. Stieg, B.C. Regan, D. C. Martin, R.B. Kaner, J. Am. Chem. Soc. 134 (2012) 9251-9262.

[22] E.A. Sanches, J.C. Soares, A.C. Mafud, E.G.R. Fernandes, F.L. Leite, Y.P. Mascarenhas, J. Mol. Struct. 1036 (2013) 121-126.

[23] W. Yang, Z. Gao, N. Song, Y. Zhang, Y. Yang, J. Wang, J. Power Sources. 272 (2014) 915-921.

[24] L. Zhang, G.I.N. Waterhouse, L. Zhang, J. Nanomater. 2011 (2011) 1-7. 